\section{Estudo numérico da influência do coeficiente de atrito no ensaio de indentação em filmes finos}

\author{
Numerical study of the influence of friction \\ coefficient for indentation testing in thin films
}

Araújo, R. ${ }^{1}$, Dias, A.M.S. ${ }^{2}$, Godoy, G.C.D. ${ }^{3}$

\author{
${ }^{1}$ Programa de Mestrado em Engenharia Mecânica - UFSJ \\ CEP: 36307-352, São João Del-Rei, MG. \\ e-mail: rodrigoaraujodivi@hotmail.com \\ ${ }^{2}$ Departamento de Engenharia Mecânica - UFRN, Natal, RN \\ e-mail: avelino.dias@ct.ufrn.br \\ ${ }^{3}$ Departamento de Engenharia Metalúrgica - UFMG, Belo Horizonte, MG \\ e-mail: gcgodoys@demet.ufmg.br
}

\section{RESUMO}

A demanda por componentes mais resistentes ao desgaste e à corrosão tem promovido um interesse crescente da engenharia de superfícies que desenvolve processos para melhoria das propriedades tribológicas de materiais. O uso de revestimentos cerâmicos tem sido uma opção utilizada para melhorar estas propriedades mecânicas superficiais. Contudo se faz necessário o desenvolvimento de ensaios para avaliação do comportamento mecânico do filme e da sua interface com o substrato, o que requer equipamentos sofisticados, mão de obra especializada, acarretando altos custos operacionais. Com o avanço dos sistemas computacionais, a utilização da análise numérica para solucionar inúmeros problemas tecnológicos tem sido cada vez mais frequente e atualmente permite a sua implementação a baixos custos de processo. Neste trabalho, propõe-se a utilização do Método dos Elementos Finitos, através de um software comercial, para simular o ensaio de indentação com penetradores esféricos em conjugados compostos de substrato metálico e filme cerâmico com diferentes espessuras. Porém a utilização de técnica numérica para avaliar o ensaio de indentação em recobrimentos superficiais finos também tem apresentado desafios, principalmente na obtenção do comportamento mecânico do conjugado. Para melhor representar o ensaio de indentação foram considerados o efeito do coeficiente de atrito entre o penetrador e o recobrimento para, em seguida, serem analisados os campos de tensões e de deformações no conjugado para diferentes profundidades de penetração.

Palavras chaves: Ensaio de Dureza, Filmes Finos, Elementos Finitos, Coeficiente de Atrito.

\section{ABSTRACT}

The demand for components more resistant to wear and corrosion has promoted a growing interest in the surface engineering that develops processes to improve the tribological properties of materials. The use of ceramic coating has been an option used to improve the surface of mechanical properties. However, it is necessary to use tests to evaluate the mechanical behavior of the film and its interface with the substrate, which requires sophisticated equipment, skilled labor and consequently expensive cost. As computer systems have been improving recently, the use of numerical analysis to solve many technological problems has been increasingly frequent and current. Consequently, it allows its implementation with a low cost. This paper proposes to use the Finite Element Method (FEM) to simulate the indentation test with spherical indenters in conjugated compounds of metal substrate and ceramic film with different thicknesses, using commercial software. However, the use of this numerical technique to evaluate the indentation testing also has had problems in obtaining the mechanical properties of the conjugate. In this work, the numerical model also includes the effects of friction between the penetrator and the film to analyze the fields of stresses and strains along the different penetration depths.

Keywords: Hardness Testing, Thin Films, Finite Element, Friction Coefficient. 


\section{INTRODUÇÃO}

A necessidade de melhorar as propriedades mecânicas como, por exemplo, a resistência à oxidação e desgaste de componentes, tem impulsionado o avanço na área de engenharia de superfícies. Esta área da engenharia trata da tecnologia de preparação e modificação das superfícies para cumprir funções específicas dentro de certa aplicação. Uma das opções utilizadas para melhorar estas propriedades é o uso de revestimentos cerâmicos. Contudo, é necessário avaliar as propriedades mecânicas destes recobrimentos e de sua interface com o substrato. O ensaio de indentação instrumentada tem sido uma técnica utilizada para avaliar as propriedades mecânicas destes conjugados.

Os ensaios de indentação foram amplamente usados para a determinação da dureza superficial em diferentes classes de materiais. Devido à sua versatilidade, numerosos trabalhos têm sido desenvolvidos neste campo, estudando-se novas metodologias e aplicações para estes ensaios. Recentes propostas utilizam estes ensaios como uma ferramenta capaz de avaliar características mecânicas dos materiais além da dureza superficial como, por exemplo, o módulo de elasticidade $(E)$, o coeficiente de Poisson $(v)$, a tenacidade à fratura $\left(K_{I C}\right)$ e uma curva de tensão em função da deformação do comportamento elastoplástico [1,2].

O ensaio de indentação instrumentada é realizado por meio de um equipamento de precisão que permite monitorar por meio de sensores as variações da profundidade de penetração $(h)$ de um penetrador em função da carga aplicada $(P)$, quando este penetra no material estudado, atingindo um deslocamento máximo e retornando para a posição inicial, completando um ciclo de carregamento e descarregamento. A Figura 1 ilustra um comportamento típico desta curva de carga em função do deslocamento, assim como, três importantes parâmetros que devem ser determinadas neste procedimento: a carga máxima de indentação $\left(P_{\max }\right)$; a profundidade máxima de penetração $\left(h_{\max }\right)$; e, o coeficiente de rigidez elástica $(S=d P / d h)$. A exatidão na obtenção destes três parâmetros influencia na determinação de valores exatos de dureza e do módulo de elasticidade. Entretanto, a implementação desta técnica de indentação para a avaliação das propriedades mecânicas e os seus resultados obtidos ainda ocasionam dúvidas no meio científico. Segundo a literatura, estes problemas são mais intensos quando se pretende avaliar o comportamento mecânico de filmes finos depositados em substratos macios [3]. Para a realização do ensaio de indentação instrumentada é necessário equipamentos de alta precisão para monitorar baixíssimas cargas e sensíveis deformações para avaliar com confiabilidade as propriedades mecânicas utilizando grandezas em micro e nanoescalas [4]. Em função destas limitações na análise dos ensaios de indentação o uso de uma técnica numérica capaz de avaliar os campos de tensões e de deformações durante o ciclo de indentação pode auxiliar em uma interpretação mais segura deste ensaio. Nas últimas décadas, esta metodologia numérica começou a ser estudada através de modelos discretos de elementos finitos para avaliar o comportamento de diferentes materiais sob o ensaio de indentação [5-9].

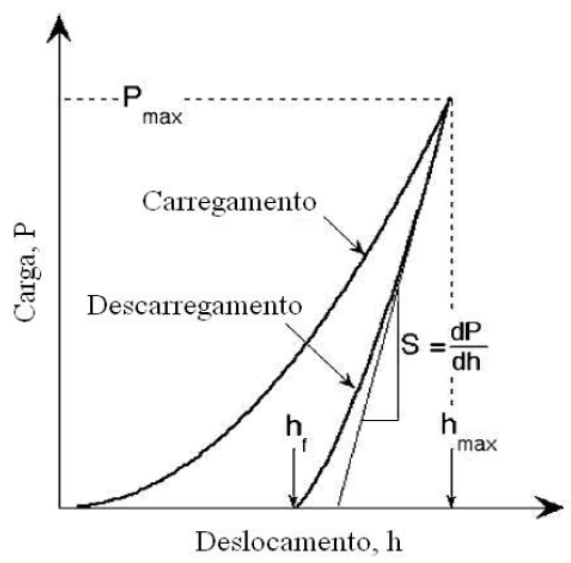

Figura 1: Ilustração esquemática de uma curva de carga do penetrador versus deslocamento mostrando parâmetros importantes de medição como o coeficiente de rigidez elástica $(S)$ e a profundidade de penetração residual $\left(h_{f}\right)$ [11].

O Método dos Elementos Finitos (MEF) tem se mostrado uma técnica numérica confiável para análise de tensões e deformações e para a simulação de diferentes problemas de engenharia. Este método tem sido largamente utilizado para simular e resolver inúmeros problemas não lineares nas áreas de instabilidade estrutural, de sistemas dinâmicos, sistemas fluidos, eletromagnéticos e de conformação mecânica. Porém, a utilização desta técnica numérica para avaliar o ensaio de indentação em recobrimentos superficiais finos 
ainda tem apresentado problemas, sejam pelas limitações computacionais ou pelas dificuldades na implementação de modelos que representem um ensaio real e, principalmente, com a devida caracterização da interface entre o filme e o substrato [2, 5-10].

A simulação proposta neste trabalho utilizará modelos discretos através do MEF, para reproduzir o ensaio de indentação com penetrador esférico, conforme ilustrado na Figura 2. O conjugado estudado foi composto de um substrato metálico recoberto por um filme cerâmico de diferentes espessuras. No procedimento numérico, avaliou-se a influencia do atrito entre o penetrador e a superfície do filme, os efeitos da espessura do recobrimento, suas propriedades de elasticidade, de resistência mecânica e o desenvolvimento do campo de tensões e deformações. Através da simulação, pretendeu-se também, prever o comportamento mecânico da interface nestes conjugados formados por recobrimentos de alta dureza depositados em substratos de média dureza.
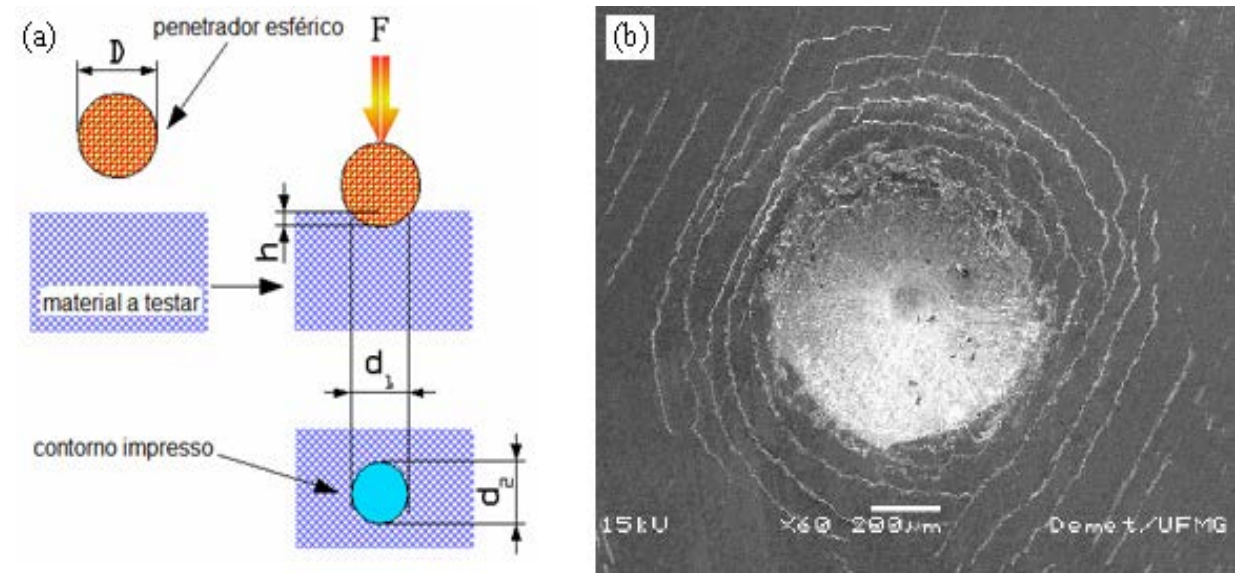

Figura 2: Ensaio de dureza Brinell: (a) representação esquemática do ensaio, sendo $D$ o diâmetro do penetrador, $h$ a profundidade de indentação, $d$ o diâmetro impresso na amostra e $F$ a força aplicada ao penetrador; $(b)$ trincas circunferenciais ao redor da impressão do penetrador esférico na superfície de uma amostra de recobrimento CrAlN [12].

\section{MATERIAIS E MÉTODOS}

A simulação numérica realizada neste trabalho utilizou o método dos elementos finitos, através do software comercial MARC ${ }^{\mathrm{TM}}$ [13], para modelar o ensaio de indentação com penetrador esférico em um filme fino cerâmico depositado sobre um substrato metálico. O modelo utilizou elementos axissimétricos, diminuindo consideravelmente os esforços computacionais [8]. Isto foi possível devido à simetria do problema. O conjugado estudado foi composto por um substrato de aço liga AISI 4140 recoberto por um filme de Nitreto de Cromo com Alumínio (CrAlN), com as seguintes espessuras: 3,0; 6,0 e 9,0 $\mu \mathrm{m}$. os materiais estudados (filme e substrato) foram considerados isotrópicos e homogêneos. Para representar o comportamento elastoplástico sob compressão destes materiais, adotou-se a curva do regime linear elástico e de escoamento plástico conforme Equação (1) descrita na literatura em Hosford e Caddell [14]. Nesta expressão, $\sigma, \varepsilon, E$ são, respectivamente, a tensão efetiva, a deformação efetiva e o Módulo de Young. $K$ e $n$ são constantes que descrevem as características de encruamento do material, sendo $n$ seu coeficiente de encruamento [15].

$$
\sigma=\left\{\begin{array}{c}
E \varepsilon^{1.0}(\text { regime elástico }) \\
\left.K \varepsilon^{n} \text { (regime plástico }\right)
\end{array}\right.
$$

Esta expressão foi reorganizada na forma da Equação (2), para ser tornar compatível com a curva de potência que permite inserir este comportamento elasto-plástico na entrada de dados do pacote de elementos finitos [12]. Nesta segunda expressão, $\sigma_{e}, \varepsilon_{e}, n$ e $\sigma_{o}$ são, respectivamente, a tensão efetiva, a deformação efetiva, o coeficiente de encruamento e o limite de escoamento. A Tabela 1 ilustra as propriedades mecânicas do filme e do substrato adotados no presente trabalho. Os valores experimentais do módulo de Young $(E)$ e do coeficiente de Poisson ( $v$ ), assim como, os dados da Equação (1) foram obtidos a partir da literatura especializada $[9,16]$.

$$
\sigma_{e}: \max \left[\left(K \varepsilon_{e}^{n}\right), \sigma_{o}\right]
$$


Tabela 1: Propriedades mecânicas do filme e do substrato [9, 16].

\begin{tabular}{l|l|l|l|l|l}
\hline MATERIAL & E (GPA) & N & IO (MPA) & K (MPA) & $n$ \\
\hline Filme (CrAlN) & 350,00 & 0,22 & 3790 & 10615 & 0,229 \\
\hline Substrato (AISI 4140) & 238,00 & 0,29 & 565 & 2230 & 0,228 \\
\hline
\end{tabular}

Simulou-se o ciclo de indentação (carregamento e descarregamento) através do deslocamento prescrito do penetrador, permitindo assim um melhor controle numérico no início e durante a simulação do ensaio [8-10]. Além desse controle numérico, o ensaio foi executado em duas fases, uma na descida do penetrador, seguida da subida do mesmo, completando-se o ciclo. Considerou-se que os conjugados estudados possuíam perfeita aderência entre o filme e o substrato. Nos modelos simulados, esta perfeita adesão foi garantida adotando-se nós coincidentes na interface entre o filme e o substrato [4]. Na análise incremental do problema foram usados cinquenta incrementos para a fase de carregamento e os mesmos cinquenta incrementos para a fase de descarregamento.

O penetrador foi modelado como uma superfície circular rígida, com diâmetro de 0,40 mm, que ensaia a amostra considerada como um material deformável, cujas propriedades simulam os conjugados estudados. Como condições de contorno do problema, o modelo possui restrição ao deslocamento na sua base e restrição ao deslocamento radial dos nós que estão localizados no eixo de simetria [17]. As características dos modelos numéricos estão ilustradas na Tabela 2.

Tabela 2: Características dos modelos numéricos axissimétricos simulados.

\begin{tabular}{l|l|l|l}
\hline CONJUGADO & ESPESSURA DO FILME & MALHA DO FILME & MALHA DO SUBSTRATO \\
\hline \multirow{3}{*}{ CrAIN - AISI 4140} & $3,0 \mu \mathrm{m}$ & 945 elementos & \\
\cline { 2 - 3 } & $6,0 \mu \mathrm{m}$ & 1890 elementos & \\
\cline { 2 - 3 } & $9,0 \mu \mathrm{m}$ & 2835 elementos & \multirow{2}{*}{7500 elementos } \\
\hline
\end{tabular}

Com o intuito de se obter uma melhor distribuição do campo de tensões e deformações na região do contato do penetrador e na interface do recobrimento com o substrato, utilizou se uma malha mais refinada nestas duas regiões. A Figura 3 ilustra este refinamento nestas regiões e também mostra as condições de contorno do problema. Foram utilizados elementos axissimétricos de quatro nós com dimensões de 0,33 $\mu \mathrm{m}$ por $1,33 \mu \mathrm{m}$ para a região mais refinada da malha.

No presente trabalho, variou-se a profundidade de penetração do ensaio em função da espessura do recobrimento. As profundidades de penetração simuladas foram de $10 \%$, 20\%, 25\% e 50\% da espessura do recobrimento. $\mathrm{O}$ objetivo principal deste trabalho foi de estudar a influência do coeficiente de atrito entre o penetrador e a superfície da amostra no comportamento mecânico do conjugado filme/substrato. Para isso, adotaram-se os seguintes valores para o coeficiente de atrito: 0,1, 0,2, 0,3 e 0,4.

\section{RESULTADOS E DISCUSSÃO}

Os gráficos das Figuras 4, 5 e 6 apresentam os resultados numéricos obtidos para a carga de indentação em função da profundidade de penetração considerando-se diferentes valores do coeficiente de atrito entre o penetrador e a amostra.

Estes resultados mostraram que numericamente a variação do coeficiente de atrito entre o penetrador e a superfície do filme apresenta pouca influência no valor da carga de indentação. Estas observações também foram feitas por Bressan et al [18] e Huang \& Pelegri [19]. Desta forma, em simulações numéricas do ensaio de indentação, pode-se desconsiderar o atrito entre o penetrador e a amostra, do ponto de vista da carga de indentação [5-10].

Os resultados também mostraram que a carga aplicada pelo penetrador foi maior em um conjugado recoberto com um filme de maior espessura do que à aplicada com mesma profundidade em um recobrimento menos espesso. Esta situação pode ser observada para o ensaio realizado no recobrimento de 3,0 $\mu \mathrm{m}$ com profundidade de penetração de $1,5 \mu \mathrm{m}$ ( $50 \%$ espessura), a carga média foi de aproximadamente $71 \mathrm{~N}$ quando comparado com os resultados obtidos para o filme de 6,0 $\mu \mathrm{m}$ com a mesma profundidade de penetração $1,5 \mu \mathrm{m}$ (25\% da espessura) onde a carga foi de aproximadamente $73 \mathrm{~N}$. Este aumento da carga pode indicar um aumento nas propriedades tribológicas do conjugado. Contudo essa variação da carga de indentação não 
foi significativa para um recobrimento com o dobro da espessura [17].

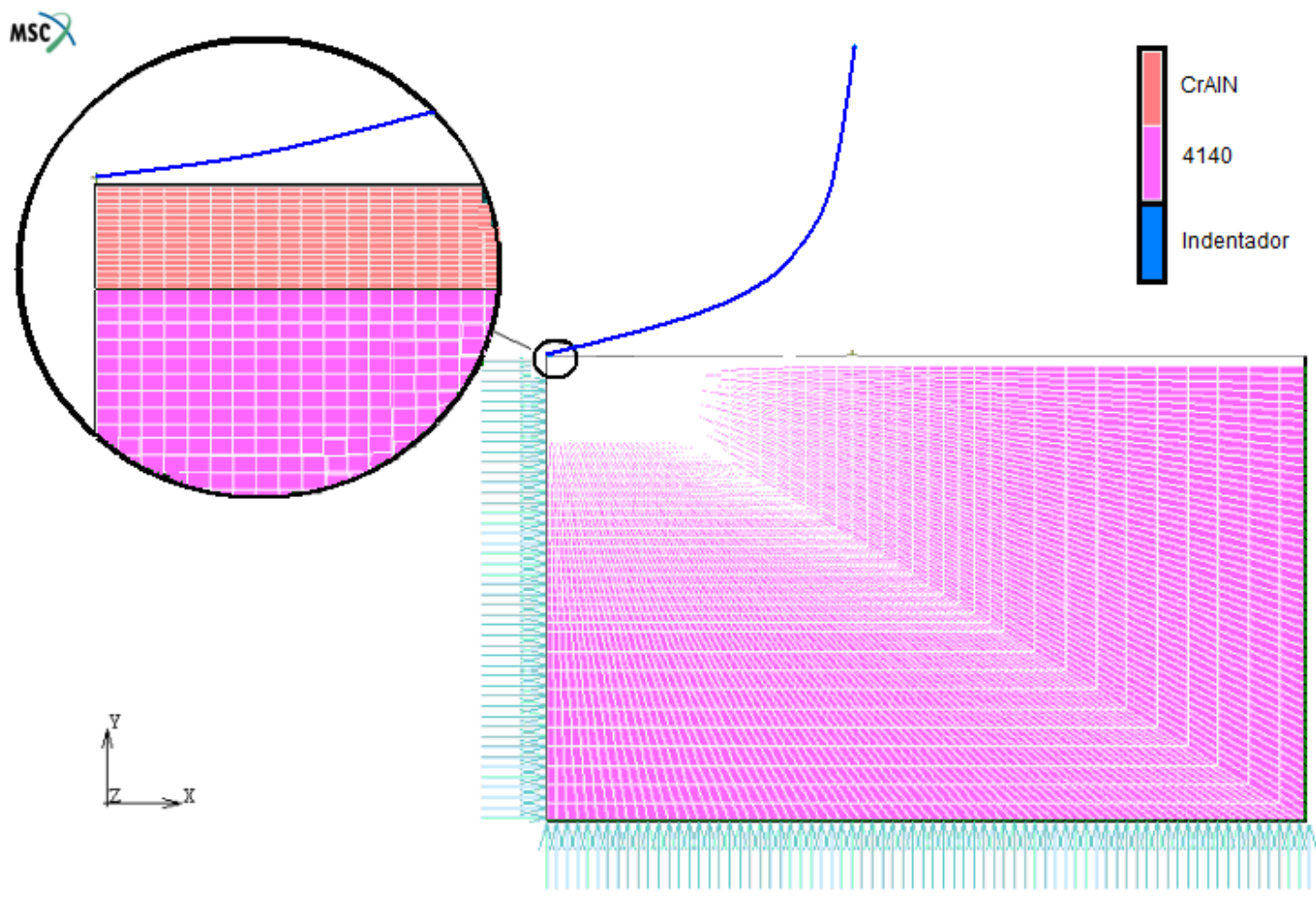

Figura 3: Modelo Numérico do ensaio Brinell em uma amostra do conjugado CrAlN-AISI 4140.

Carga de Indentação para conjugado com filme de $3 \mu \mathrm{m}$ de espessura

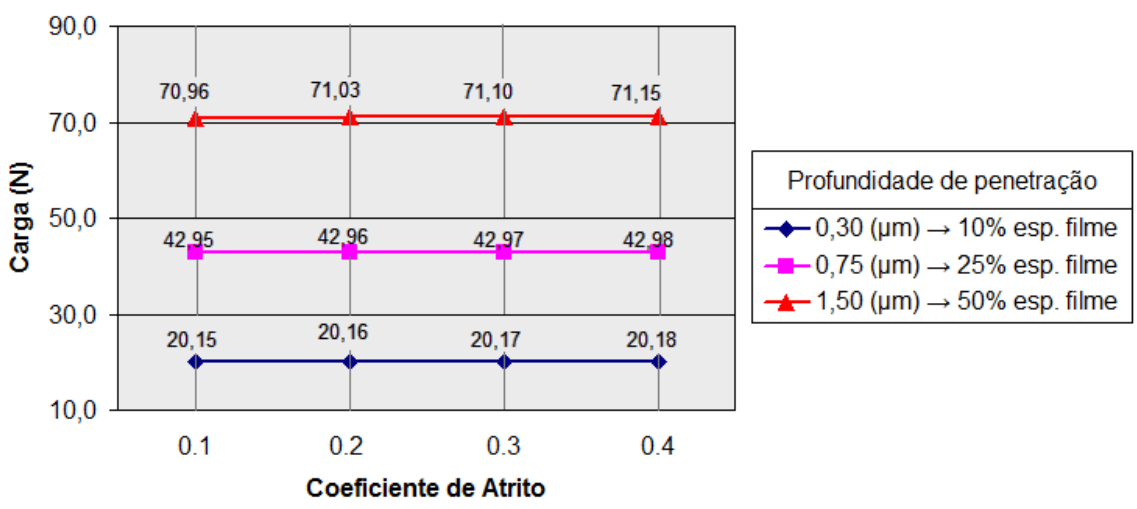

Figura 4: Carga em função do atrito entre o penetrador e a superfície do filme com 3,0 $\mu \mathrm{m}$ de espessura.

Na sequencia, avaliou-se o comportamento numérico do campo de tensões durante o ensaio de indentação em função da variação do coeficiente de atrito entre o penetrador e a amostra. Foram escolhidas três regiões no filme para ilustrar o comportamento destas tensões durante o ciclo de indentação, Figura 7. A primeira região escolhida foi o ponto de contato central do penetrador com a amostra. A segunda foi próxima a interface entre o filme e o substrato, diretamente abaixo do contato central. A terceira região foi na superfície, próximo a borda da impressão da indentação, mas fora da marca de indentação. Neste trabalho, a escolha desta ultima região foi por ela apresentar trincas circunferenciais, durante o ensaio de indentação real em filmes de CrAlN (Figura 2). Esta Figura 7 também ilustra os três nós escolhidos nas regiões acima descritas para o modelo numérico com filme de espessura de 3,0 $\mu \mathrm{m}$, considerando uma profundidade de penetração de vinte por cento de sua espessura $(0,6 \mu \mathrm{m})$. 


\section{Carga de Indentação para conjugado com filme de $6 \mu \mathrm{m}$ de espessura}

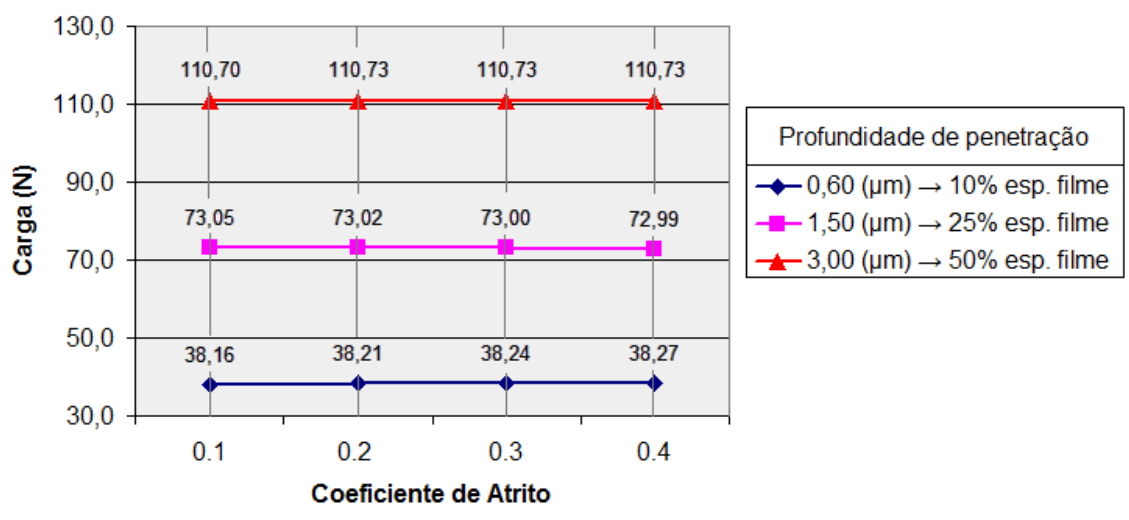

Figura 5: Carga em função do atrito entre o penetrador e a superfície do filme com 6,0 $\mu \mathrm{m}$ de espessura.

\section{Carga de Indentação para conjugado com filme de $9 \mu \mathrm{m}$ de espessura}

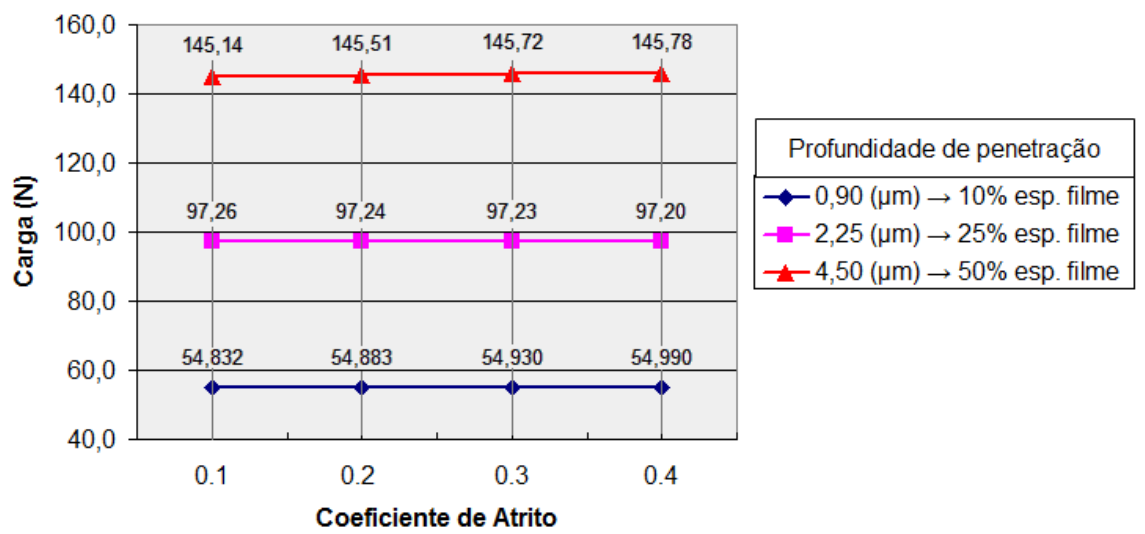

Figura 6: Carga em função do atrito entre o penetrador e a superfície do filme com 9,0 $\mu$ m de espessura.

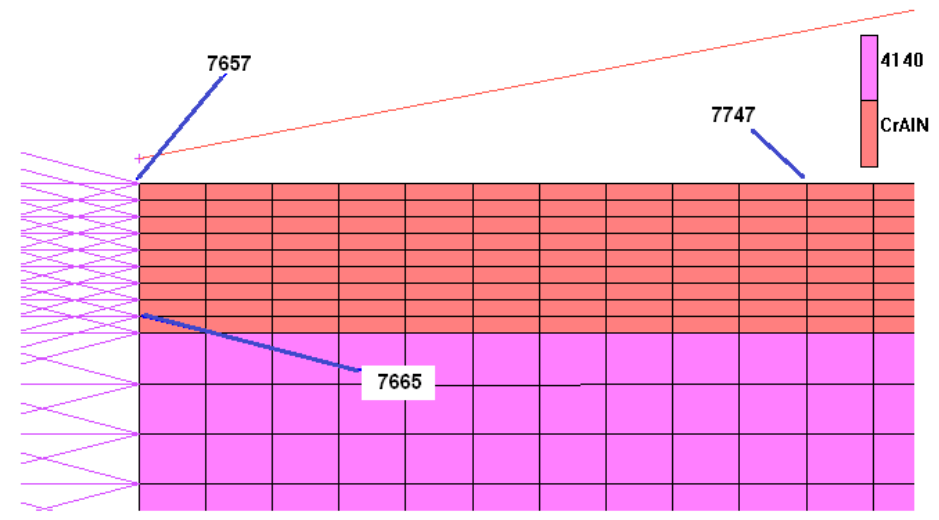

Figura 7: Posição dos nós na região de contato (7657), próximo a interface (7665) e na superfície da amostra (7747) para modelo numérico do ensaio de indentação com filme de 3,0 $\mu \mathrm{m}$.

A Figura 8 mostra o comportamento numérico da Máxima Tensão Principal $\left(\sigma_{1}\right)$ na região de contato entre o penetrador e a amostra durante o ciclo de indentação. Estas análises consideraram uma profundidade de penetração de vinte por cento da espessura do filme. Os resultados mostraram que o comportamento desta tensão nesta região se mantém praticamente inalterado durante o ciclo do ensaio considerando diferentes 
coeficientes de atrito entre o penetrador e a amostra. Há uma pequena variação do valor desta tensão em função do coeficiente de atrito quando o penetrador está praticamente no final da fase de carregamento (incremento 40). Porém, nesse instante, esta diferença foi de $10 \%$ entre as Máximas Tensões Principais considerando o atrito igual a zero e o coeficiente de atrito de 0,4.

A Figura 9 mostra o comportamento da Máxima Tensão Principal $\left(\sigma_{1}\right)$ na região da interface entre o filme de 3,0 $\mu \mathrm{m}$ e o substrato durante o ciclo de indentação. Nesta região, verifica-se que o comportamento numérico desta Tensão Principal cresce em função do aumento do coeficiente de atrito entre o indentador e a amostra. Neste caso, ao final da fase de carregamento do ensaio, a diferença entre a Máxima Tensão Principal considerando o atrito igual a zero e a Tensão Principal para o atrito de 0,4 foi de $23 \%$. Estes resultados numéricos indicam que, com o aumento do atrito entre o penetrador e a amostra, ocorre uma redistribuição no campo de tensões no filme. Em particular, para este filme com 3,0 $\mu \mathrm{m}$ de espessura, o aumento deste atrito acarreta um crescimento na Máxima Tensão Principal na região da interface entre o filme e o substrato tornando essa região mais critica do ponto de vista desta tensão.

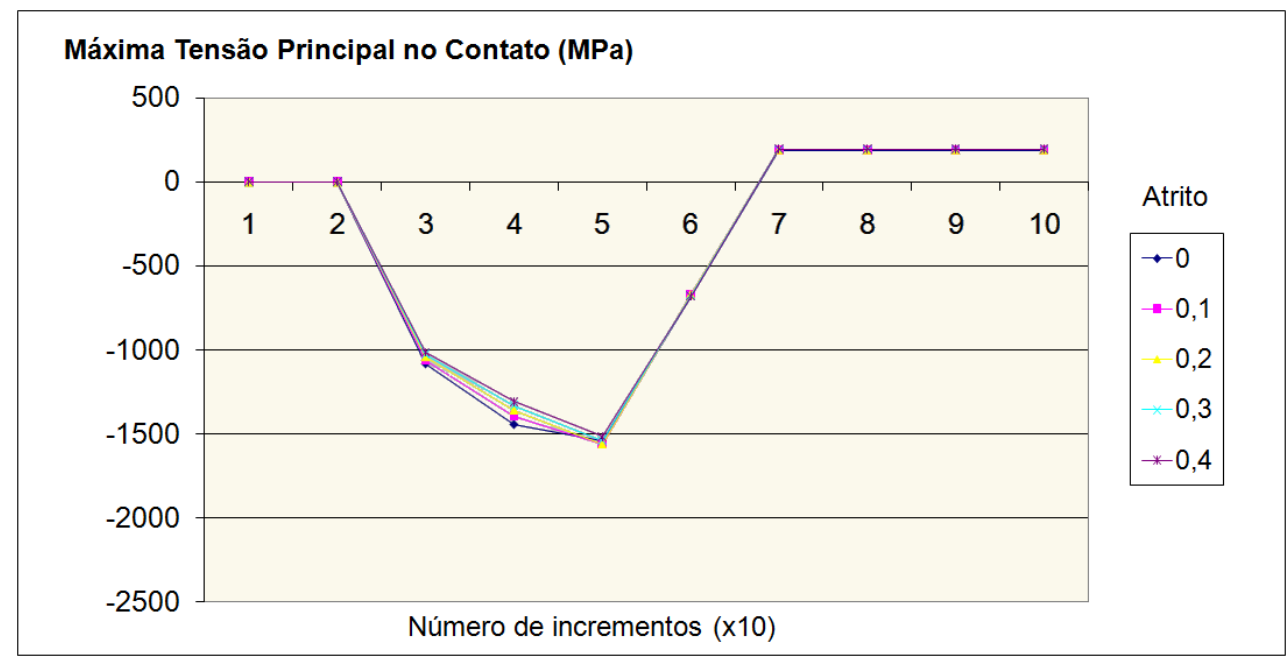

Figura 8: Comportamento da Máxima Tensão Principal no contato central (nó 7657) para o ensaio de indentação com filme de 3,0 $\mu$ m e profundidade de penetração de $20 \%$ da espessura do filme.

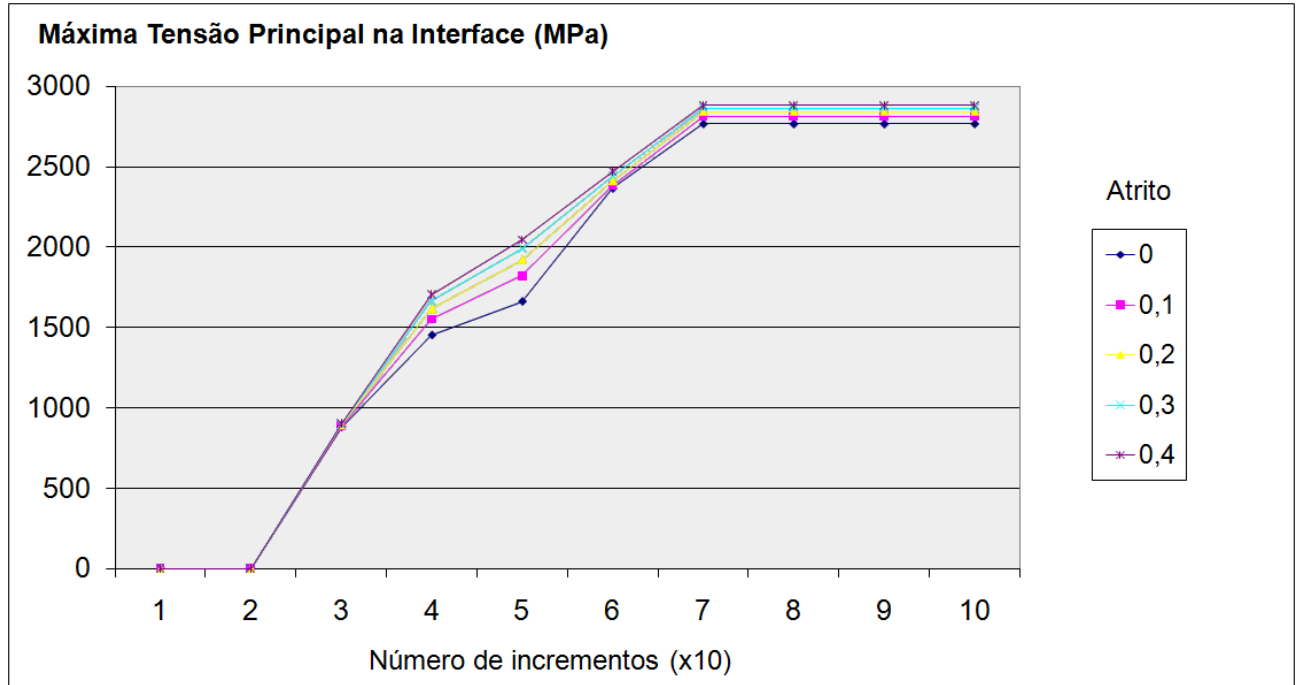

Figura 9: Comportamento da Máxima Tensão Principal na interface entre o filme e o substrato (nó 7665). Filme de 3,0 $\mu \mathrm{m}$ e profundidade de penetração de $20 \%$ da espessura do filme.

A Figura 10 mostra o comportamento da Máxima Tensão Principal $\left(\sigma_{1}\right)$ na superfície da amostra durante o ciclo do ensaio, na região da borda da indentação, mas fora da marca da impressão. Há uma variação desta tensão em função do coeficiente de atrito quando o penetrador está praticamente no final da 
fase de carregamento (incremento 40). Porém, nesse instante, a diferença entre a Máxima Tensão Principal considerando o atrito igual a zero e a Tensão Principal para o atrito de 0,4 foi de apenas 6,6\%.

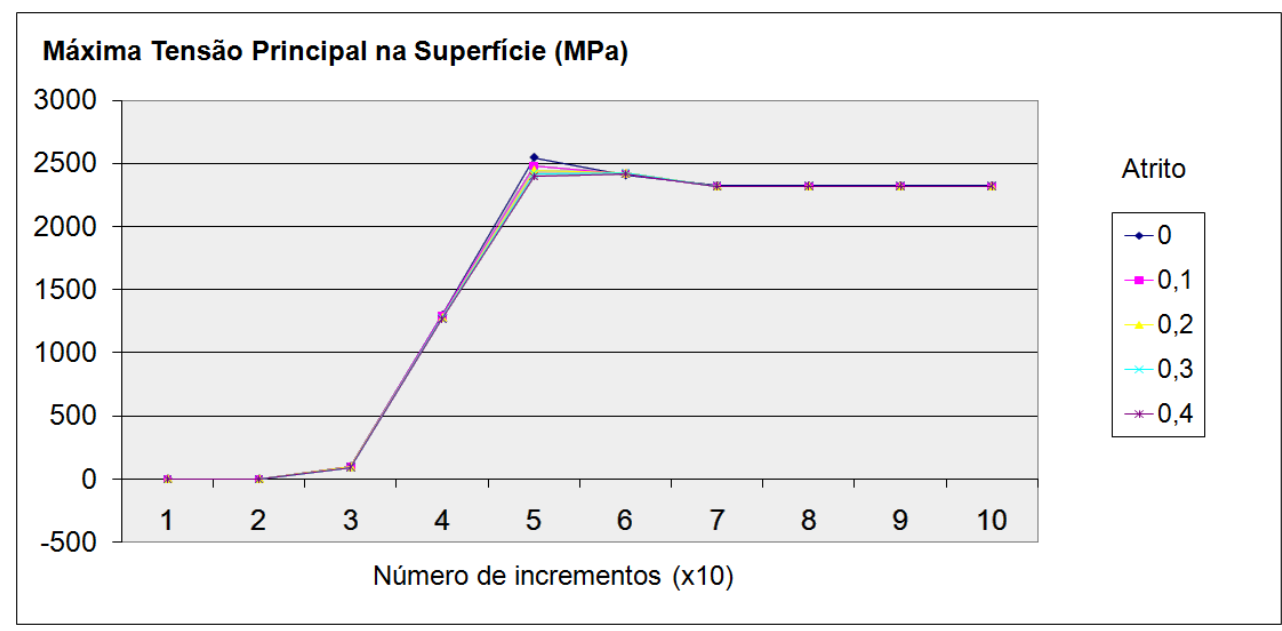

Figura 10: Comportamento da Máxima Tensão Principal na borda da marca da indentação (nó 7747). Filme de 3,0 $\mu \mathrm{m}$ e profundidade de penetração de $20 \%$ da espessura do filme.

Estes primeiros resultados numéricos mostraram que o comportamento da Máxima Tensão Principal indica pequenas variações durante o ciclo do ensaio para o conjugado com filme com espessura de 3,0 $\mu \mathrm{m}$, considerando diferentes coeficientes de atrito entre o penetrador e a amostra e, considerando ainda, uma profundidade de penetração do indentador de $20 \%$ da sua espessura. Exceto para a região de interface considerada (nó 7665), que apresentou um crescimento em função do aumento do coeficiente de atrito entre o indentador e a amostra. Contudo, em todas estas análises, a amplitude destas Máximas Tensões Principais não ultrapassou o limite de escoamento considerado para o filme de CrAlN (Tabela 1). Em outras palavras, para a profundidade de penetração considerada, este filme de CrAlN está submetido a um campo de tensões e de deformações majoritariamente elástico.

Para analisar uma distribuição elasto-plástica deste campo de tensões durante o ciclo de indentação nos conjugados estudados, optou-se por implementar simulações considerando uma profundidade de penetração de cinquenta por cento da espessura do filme. A Tabela 3 ilustra os maiores valores das tensões radiais $\left(\sigma_{x x}\right)$, axiais $\left(\sigma_{y y}\right)$ e Máximas Tensões Principais $\left(\sigma_{1}\right)$ para as três regiões anteriormente especificadas, ou seja, a região do contato, da interface e da borda da marca da indentação. Foram comparados os resultados considerando atrito igual a zero e de 0,4 entre o indentador e a amostra. Na tabela, o número entre parêntesis ao lado do valor da tensão indica em qual interação ocorreu esse valor da tensão. Também foram comparados os resultados para conjugados com duas diferentes espessuras, de 3,0 $\mu \mathrm{m}$ e de 6,0 $\mu \mathrm{m}$, respectivamente.

Inicialmente, verificou-se que a magnitude das tensões normais na direção radial $\left(\sigma_{x x}\right)$ e da Máxima Tensão Principal $\left(\sigma_{1}\right)$ no contato central diminui com crescimento do valor do coeficiente de atrito. Este comportamento para estas tensões nesta região também foi observado por outros trabalhos encontrados na literatura [15]. Entretanto, houve um aumento na magnitude das tensões axiais com o aumento do atrito. Isto provavelmente ocorreu devido às restrições ao deslocamento superficial ocasionado pela imposição do atrito entre o indentador e a amostra. Em seguida, observou-se que há um pequeno aumento no nível das tensões na interface em função do crescimento do coeficiente de atrito. Percebeu-se também que, neste caso, estes maiores valores ocorreram durante o descarregamento do ensaio. Em contrapartida, ocorreu um decrescimento na magnitude destas tensões com o aumento da espessura do filme. Já as magnitudes das tensões na borda da impressão do indentador diminuem com o crescimento do coeficiente de atrito. Nesta ultima região, tanto para as análises com atrito zero, quanto para as análises considerando um atrito de 0,4, a magnitude das tensões radiais e das Máximas Tensões Principais apresentou pequenas variações.

Estes resultados numéricos obtidos para o campo de tensões na região da borda da marca de indentação também mostrou que esta é uma região crítica do ponto de vista das tensões radiais e das Máximas Tensões Principais. Inclusive, considerando os resultados obtidos para as maiores profundidades de penetração do indentador, a magnitude das tensões $\sigma_{x x}$ e $\sigma_{1}$ ultrapassa o limite de escoamento aqui considerado para o filme CrAlN (Tabela 1). Estes resultados se mostraram coincidentes com as observações 
feitas por Begley et. al. (1999), apesar de esses autores focarem suas análises no campo de deformações radiais na borda da impressão, porém dentro da marca de indentação [15]. Mas, ocorreu uma convergência entre os resultados numéricos com os obtidos experimentalmente, uma vez que, no ensaio experimental de indentação em filmes de CrAlN depositados em substrato metálico de alta dureza (aço AISI 4140), esta região apresenta trincas circunferenciais ao final do ensaio (Figura 2) [11].

Tabela 3: Resultados numéricos para as tensões radiais $\left(\sigma_{x x}\right)$, axiais $\left(\sigma_{y y}\right)$ e Máximas Tensões Principais $\left(\sigma_{1}\right)$, nas regiões do contato central, da interface entre o filme e o substrato e da borda fora da marca de indentação. $\mathrm{O}$ valor entre parêntesis representa o numero do incremento em que ocorreram estas tensões.

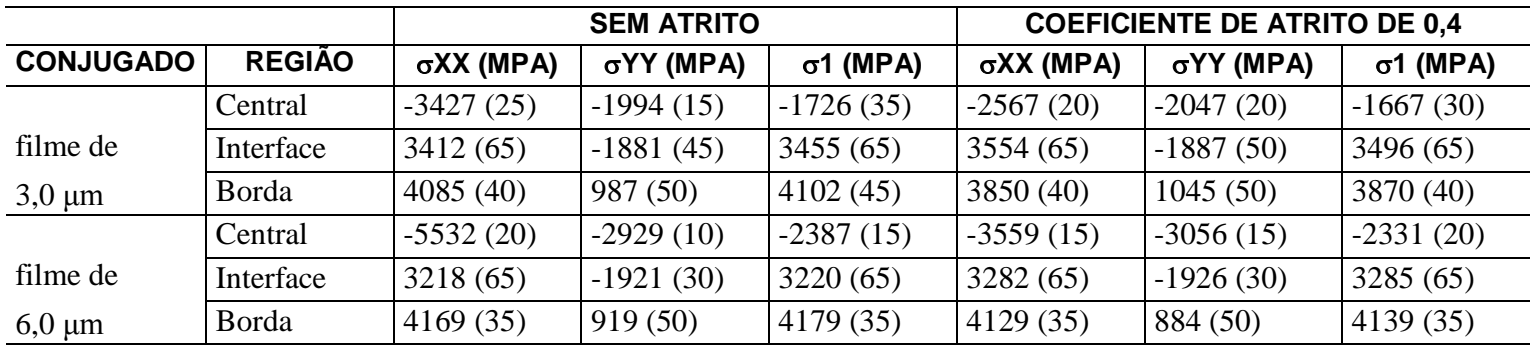

Continuando com a comparação entre os resultados obtidos para o comportamento do campo de tensões em conjugados com diferentes espessuras do filme e com diferentes coeficientes de atrito entre o indentador e a amostra, a Tabela 4 mostra a distribuição das tensões de cisalhamento $\left(\tau_{12}\right)$ no filme em quatro diferentes regiões. Repetiram-se as três regiões anteriormente discriminadas, ou seja, a região do contato central, a região de interface abaixo do contato central (interface 1) e a região na borda fora da marca da indentação (Figura 7). A quarta região escolhida se situa na interface, porém abaixo da borda da marca da indentação (interface 2). A avaliação desta tensão de cisalhamento nesta quarta região foi motivada pelo fato que a mesma tem sido associada à delaminação do filme em trabalhos publicados na literatura científica [15].

Avaliando estes resultados numéricos obtidos para esta tensão de cisalhamento $\left(\tau_{12}\right)$ no contato central, verifica-se que seus valores máximos se mantêm praticamente inalterado independente do aumento no coeficiente de atrito e da espessura do filme. Observa-se também que o comportamento desta tensão nessa região praticamente não muda durante o ciclo de indentação. Para a região da interface abaixo do contato central (interface 1), esta tensão de cisalhamento também não parece sofrer grande influência da variação do coeficiente de atrito. Porém, a magnitude desta tensão diminui em função do aumento da espessura do filme. Ocorreu um comportamento peculiar no comportamento de $\tau_{12}$ na região da marca da indentação. Para o filme com espessura de 3,0 $\mu \mathrm{m}$ houve um aumento nesta tensão em função do aumento do atrito. Por outro lado, para o filme com espessura de 6,0 $\mu \mathrm{m}$, a magnitude desta tensão de cisalhamento teve uma leve redução. Inclusive, parece não existir influência da espessura do filme nesta tensão de cisalhamento quando se considera o atrito igual a zero. Na quarta região (interface 2), houve um aumento significativo nesta tensão de cisalhamento em função do aumento do coeficiente de atrito. Este aumento na tensão de cisalhamento não ocorre com a mesma intensidade quando se simulou o ensaio em um filme com 6,0 $\mu \mathrm{m}$ de espessura.

Estes outros resultados numéricos mostram que a região da interface também se apresenta critica para a tensão de cisalhamento $\left(\tau_{12}\right)$. Seja na posição abaixo do contato central (interface 1), assim como na posição abaixo da borda da marca da impressão (interface 2). Nelas, verificou-se um aumento no campo de tensões $\left(\sigma_{x x}, \sigma_{1} \mathrm{e} \tau_{12}\right)$ com o crescimento do coeficiente de atrito na superfície da amostra. Estes resultados parecem indicar que, para conjugados de mesma espessura, o aumento do atrito pode favorecer um processo de delaminação do filme. Por outro lado, também se observou que o aumento deste atrito não acarreta grandes variações neste campo de tensões para filmes com grandes espessuras. Ou seja, filmes de maiores espessuras apresentaram uma menor possibilidade de delaminação, uma vez que, a distribuição numérica do campo de tensões se mantém praticamente inalterada na interface com o substrato [5, 17]. 
Tabela 4: Resultados numéricos para as tensões de cisalhamento $\left(\tau_{12}\right)$ nas regiões do contato central, da interface entre o filme e o substrato (em baixo do contato central), da borda fora da marca de indentação e da interface em baixo da marca de indentação.

\begin{tabular}{l|l|l|l}
\hline \multicolumn{2}{l}{} & SEM ATRITO & COEFICIENTE DE ATRITO DE 0,4 \\
\hline CONJUGADO & REGIÃo & $\tau 12$ (MPA) & $\tau 12$ (MPA) \\
\hline & Central & $471(15-60)$ & $476(15-50)$ \\
\cline { 2 - 4 } filme de 3,0 $\mu \mathrm{m}$ & Interface (1) & $519(20)$ & $493(20)$ \\
\cline { 2 - 4 } & Borda & $377(50)$ & $511(55)$ \\
\cline { 2 - 4 } & Interface (2) & $760(45)$ & $904(50)$ \\
\hline \multirow{4}{*}{ filme de 6,0 $\mu \mathrm{m}$} & Central & $473(10-60)$ & $475(10-60)$ \\
\cline { 2 - 4 } & Interface (1) & $337(15)$ & $301(15)$ \\
\cline { 2 - 4 } & Borda & $388(50)$ & $378(45)$ \\
\cline { 2 - 4 } & Interface (2) & $921(50)$ & $918(50)$ \\
\hline
\end{tabular}

Por fim, a Figura 11 mostra o comportamento numérico da Máxima Tensão Principal $\left(\sigma_{1}\right)$ na região da interface contato entre o filme e o substrato durante o ciclo de indentação, para o modelo com filme de 9,0 $\mu \mathrm{m}$ de espessura. Novamente, nesta simulação, verifica-se que o comportamento numérico desta Tensão Principal praticamente não varia com o aumento do coeficiente de atrito entre o penetrador e a amostra.

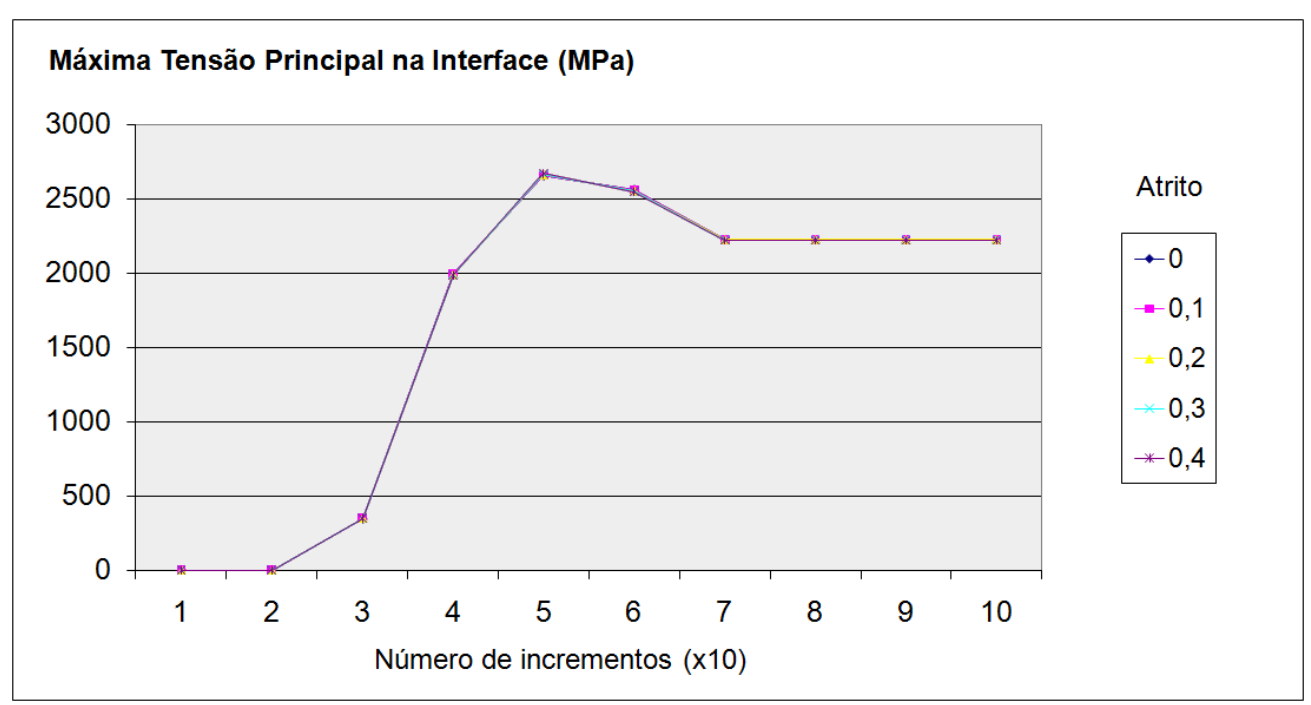

Figura 11: Comportamento da Máxima Tensão Principal na interface entre o filme e o substrato abixo do contato central para o ensaio de indentação do conjugado com filme de 9,0 $\mu \mathrm{m}$.

\section{CONCLUSÕES}

Baseado nos resultados numéricos do ensaio de indentação com penetradores esféricos através do método de elementos finitos, foi possível concluir que do ponto de vista do comportamento global, os modelos conseguiram representar o ensaio de indentação em diferentes conjugados compostos por um filme duro (CrAlN), com diferentes espessuras, depositados em um substrato metálico (AISI 4140).

Verificou-se em todas as situações simuladas que o atrito entre o penetrador e a superfície do filme não apresenta influência significativa na carga de indentação. Esta análise também mostrou que filmes de maiores espessuras proporcionaram um aumento médio de 2,7\% na carga de indentação considerando a mesma profundidade de penetração em um conjugado com o dobro da espessura do filme.

Apesar de não ter sido avaliado quantitativamente o comportamento da superfície de contato em função da variação do coeficiente de atrito, observou-se que houve uma restrição ao deslocamento radial (direção x) devido ao aumento do atrito. Estas observações também foram relatadas e discutidas em diferentes trabalhos encontrados na literatura [15, 20]. Apesar disso, alguns autores desconsideram esses efeitos uma vez que não introduzem o atrito em seus modelos numéricos [8-10, 17]. 
No presente trabalho não foi possível tirar grandes conclusões do comportamento do campo de tensões na região do contato central (7657) do indentador com o filme. Exceto aquelas que parecem óbvias como, por exemplo, o aumento na magnitude do campo de tensões axiais $\left(\sigma_{y y}\right)$ em função de uma maior da profundidade de penetração do indentador. Ou ainda, uma diminuição no campo de tensões axiais $\left(\sigma_{x x}\right)$ devido ao aumento do coeficiente de atrito, impedindo o deslocamento radial da superfície [15].

Em termos de comportamento global, o atrito altera a área de contato e o campo de tensões sob o indentador. Os resultados para os campos de tensões indicam que, com o aumento do coeficiente de atrito, ocorreu uma redistribuição das tensões provenientes da ação do indentador na amostra. Contudo, com o aumento da espessura do filme, as variações no campo de tensões ficaram restritas ao filme, não acarretando grandes variações nas tensões na sua interface com o substrato. Em outras palavras, um conjugado com filme de maior espessura pode apresentar uma menor tendência de apresentar um maior nível de tensões na interface, diminuindo uma possível delaminação em serviço. Na literatura, também é possível verificar essa tendência de que com o aumento da espessura do filme menor há uma menor a possibilidade de sua delaminação, seja através da observação numérica do campo de deformações plásticas ou do campo das deformações de trincamento durante o ciclo do ensaio [5, 17].

Em uma ultima análise, corroborando com as conclusões do trabalho de Mata e Alcalá [20], sugere-se utilizar um lubrificante na superfície da amostra para minimizar indesejáveis efeitos no campo de tensões devido ao atrito. E que esta lubrificação também seja estendida para componentes mecânicos recobertos com filmes como, por exemplo, as pastilhas de ferramentas de corte, quando em serviço.

Por fim, ainda existe a necessidade de um maior aprofundamento na análise numérica e experimental para se introduzir um parâmetro capaz de modelar de forma mais representativa a adesão entre o filme e o substrato. Como sugestão para trabalhos futuros propõe-se introduzir nesta interface uma fina camada de elementos que seja capaz de representar numericamente desde uma adesão perfeita até um processo de delaminação do filme.

\section{AGRADECIMENTOS}

Os autores agradecem ao suporte financeiro provido pela agência estadual de fomento à pesquisa FAPEMIG.

\section{BIBLIOGRAFIA}

[1] ZENG, K., CHIU, C-h., “An analysis of load-penetration curves from instrumented indentation”, Acta Materialia, v. 49, pp. 3539-3551, 2001.

[2] LEE, H., LEE, J.H., PHARR, G.M., "A numerical approach to spherical indentation techniques for material property evaluation”, Journal of the Mechanics and Physics of Solids, v. 53, pp. 2073-2069, 2005.

[3] FISCHER-CRIPPS, A.C., "Critical review of analysis and interpretation of nanoindentation test data", Surface \& Coatings Technology, v. 200, pp. 4153-4165, 2006.

[4] HUANG, X., PELEGRI, A.A., "Mechanical characterization of thin film materials with nanoindentation measurements and FE analysis”, Journal of Composite Materials, v. 40, pp. 1393-1407, 2005.

[5] SUN, Y., BLOYCE, A., BELL, T., "Finite element analysis of plastic deformation of various TiN coating/substrate systems under normal contact with a rigid sphere”, Thin Solid Films, v. 271, pp. 122-131, 1995.

[6] SOUZA, R.M., MUSTOE, G.G.W., MOORE, J.J., "Finite element modeling of the stresses, fracture and delamination during the indentation of hard elastic films on elastic-plastic soft substrates", Thin Solid Films, v. 392, n.1, pp. 65-74, 2001.

[7] ANTUNES, J.M., MENEZES, L.F., FERNANDES, J.V., "3-dimensional numerical simulation of Vickers indentation testing”, International Journal of Solids and Structures, v. 43, pp. 784-806, 2006.

[8] DIAS, A.M.S., MODENESI, P.J., CRISTINA, G.C., "Computer simulation of stress distribution during Vickers hardness testing of WC-6Co”, Materials Research, v. 9, n. 1, pp. 73-76, 2006.

[9] DIAS, A.M.S., GODOY, G.C.D, "Determination of stress-strain curve through Berkovich indentation testing”, Materials Science Forum, v. 636-637, pp. 1186-1193, 2010.

[10] PULECIO, S.A.R., FARIAS, M.C.M., SOUZA, R.M, "Finite element and dimensional analysis algorithm for the prediction of mechanical properties of bulk materials and thin films", Surface and Coatings Technology, v. 205, n. 5, pp. 1386-1392, 2010. 
[11] OLIVER, W.C., PHARR, G.M., "Measurement of hardness and elastic modulus by instrumented indentation: advances in understanding and refinements to methodology", Journal of Materials Research, v. 19, pp. 3-20, 2004.

[12] MANCOSU, R.D., Recobrimento tribológico $\mathrm{Cr}-\mathrm{N}$ e nitretação a plasma para melhoria da resistência à erosão cavitacional de um aço carbono ABNT 1045: uma abordagem topográfica, Tese de D.Sc., Escola de Engenharia da Universidade Federal de Minas Gerais, Belo Horizonte, MG, Brasil, 2005.

[13] MSC Software-MARCTM, Volume A: Theory and User Information. Users Manual, 2007.

[14] HOSFORD, W.F., CADDELL, R.M., Metal Forming, Prentice Hall, Inc., New Jersey, 363p, 1993.

[15] BEGLEY, M.R., EVANS, A.G., HUTCHINSON, J.W., "Spherical impression of thin films on elasticplastic substrates”, International journal of Solids and structures, v. 36, pp. 2773-2788, 1999.

[16] Matweb, www.matweb.com.br . Acessado em janeiro de 2011.

[17] DIAS, A.M.S., SOTANI, P.F.B., GODOY, G.C., "Simulação do ensaio de indentação em filmes finos com o uso de modelos de trinca difusa”, Revista Matéria, v. 15, n. 3, pp. 422-430, 2010.

[18] BRESSAN, J.D., TRAMONTIN, A., ROSA, C., "Modeling of nanoindentation of bulk and thin film by finite element method", Wear, v. 258, pp. 115-122, 2005.

[19] HUANG, X., PELEGRI, A.A., "Finite element analysis on nanoindentation with friction contact at the film/substrate interface”, Composites Science and Technology, v. 67, pp. 1311-1319, 2007.

[20] MATA, M., ALCALÁ, J., “The role of friction on sharp indentation”, Journal of the Mechanics and Physics of Solids, v.52, n. 1, pp. 145-165, 2004. 